\title{
Comportamiento de las Complicaciones en Adolescentes Embarazadas en la E.S.E Hospital la Divina Misericordia Magangué Bolívar, Durante el Año 2015.
}

\author{
Wendy Luz Gómez Mejía ${ }^{1}$, Ely Cala Ramos ${ }^{2}$, Katherine Guerra Hernández ${ }^{3}$, Walter Torres ${ }^{4}$. \\ ${ }^{-2}$ Bacterióloga Clínica. Especialista. en Epidemiología. Bogotá, Colombia. \\ ${ }^{3}$ Enfermera, Especialista en Epidemiología. Secretaría de Salud Municipal Magangué. Bolívar, Colombia. \\ ${ }^{4}$ Médico Especialista en Epidemiología. DADIS Cartagena, Colombia.
}

\begin{abstract}
RESUMEN
Introducción. El embarazo en adolescentes es una condición que mundialmente va en aumento; se considera que esto se debe a múltiples factores entre los cuales se podría mencionar: la constitución de la familia, las condiciones sociales y culturales y de los medios de comunicación. Las tasas de fertilidad adolescente en la región de América Latina y el Caribe no han descendido en la misma proporción que las de nivel global. De hecho, la División de Población del Departamento de Asuntos Económicos y Sociales de Naciones Unidas prevé que serán las más altas del mundo y se mantendrán estables entre 2020 y 2021 (1).
\end{abstract}

Objetivo: identificar las complicaciones frecuentes y los factores de riesgo en las adolescentes embarazadas, en el ESE Hospital la Divina Misericordia Magangué bolívar, año 2015.

Materiales y Métodos: como muestra, se tomaron 30 gestantes adolescentes, de un total de 484, mediante el análisis de historias clínicas, el cual incluyó mediciones antropométricas (peso, talla, edad,) y determinación del estado nutricional.

Resultados: Los 30 casos evidenciaron complicaciones durante su gestación. Dos casos con un 6,7\% correspondieron a adolescentes tempranas, mientras que 28 casos $93,3 \%$ fueron adolescentes tardías.

Conclusiones: Las complicaciones obstétricas como la anemia severa con 14 casos, $58,3 \%$ evidencia un alto impacto en el estrato I de la población de estudio.

Palabras clave: Embarazo, Complicaciones, Adolescentes, complicaciones en el embarazo, Mortalidad Materna.

\section{ABSTRACT}

Complications behavior in Pregnant Adolescents in the E.S.E Hospital la Divina Misericordia Magangué Bolivar, During 2015.

Introduction. Teenage pregnancy is a condition that is increasing worldwide; It is considered that this is due to multiple factors the constitution of the family, social and cultural conditions and the media. Adolescent fertility rates in the Latin American and Caribbean region have not decreased by the same proportion as those at the global level. In fact, the Population Division of the United Nations Department of Economic and Social Affairs expects them to be the highest in the world and will remain stable between 2020 and 2021 (1).

Objective: to identify frequent complications and risk factors in pregnant adolescents, E.S.E Hospital la Divina Misericordia Magangué Bolívar, 2015.

Materials and Methods: A total of 30 teenage pregnant women of 484 were taken as a sample, through the analysis of medical records, which included anthropometric measurements (weight, height, age,) and determination of nutritional status.

Results: The 30 cases showed complications during pregnancy.

Two cases with $6,7 \%$ corresponded to early adolescents, while 28 cases $93,3 \%$ were late adolescents.

Conclusions: Obstetric complications such as severe anemia with 14 cases $58,3 \%$ show a high impact on stratum I of the study population.

Keywords: Pregnancy, Complications, Adolescents, pregnancy complications, Maternal Mortality.

*Autor de correspondencia: Wendy Luz Gómez Mejía wengomezm16@gmail.com

Como citar: Gómez Mejía, Wl, Cala Ramos, E, Guerra Hernández, K, Torres, W. Comportamiento de las Complicaciones en Adolescentes Embarazadas en la E.S.E Hospital la Divina Misericordia Magangué Bolivar, Durante el Año 2015. Revista

Cuarzo 2019;25(1):11-15

Recibido: 22 de noviembre de 2018 Aceptado: 4 de febrero de 2019 Publicado: 30 de junio de 2019

DOI: https://doi.org/10.26752/cuarzo.v25.n1.320 


\section{INTRODUCCIÓN}

$\mathrm{E}$ 1 embarazo en adolescentes es un asunto complejo con muchas razones para preocuparse. Los niños de 12 a 14 años de edad son más propensos que otros adolescentes a tener relaciones sexuales no planeadas y de ser convencidos a tener sexo (2).

Los factores de riesgo para el embarazo en adolescentes abarcan: edad más joven, rendimiento académico deficiente, desventaja económica, padres solteros adolescentes.

A nivel mundial, según el Fondo de Naciones Unidas para la Infancia (UNICEF), cada año dan a luz 15 millones de adolescentes, entre los 15 y 19 años de edad, en todo el mundo. el 95\% de éstos, ocurre en países en desarrollo (3).

Las adolescentes menores de 16 años corren un riesgo de defunción materna cuatro veces más alto que las mujeres de 20 a 30 años, y la tasa de mortalidad de sus neonatos es aproximadamente un $50 \%$ superior. Los expertos en salud convienen en que las adolescentes embarazadas requieren atención física y psicológica especial durante el embarazo, el parto y el puerperio para preservar su propia salud y la de sus recién nacidos (4).

Se estima que 16 millones de niñas de edades comprendidas entre los 15 y los 19 años dan a luz cada año, y un $95 \%$ de esos nacimientos se producen en países en desarrollo, según el estudio realizado por Rosen. Esto representa el $11 \%$ de todos los nacimientos en el mundo. Sin embargo, los promedios mundiales ocultan importantes diferencias regionales (5).

Adolescente temprana: Es la primera etapa de desarrollo del niño. Se caracteriza por los cambios físicos a una gran velocidad. En esta fase aparecen los caracteres sexuales secundarios, de 10 a 14 años.

Adolescente tardía: El adolescente ya es físicamente adulto y sus órganos sexuales están plenamente capacitados para la reproducción, de 15 a 19 años.

\section{METODOLOGÍA}

Tipo de estudio: estudio descriptivo retrospectivo.

Población en estudio: Las muestras para este estudio se tomaron de 30 gestantes adolescentes a través del análisis individual de historias clínicas, atendidas en la E.S.E Divina Misericordia de Magangué - Bolívar y municipios aledaños.

El estudio se realizó durante el año 2015, de las historias clínicas se extrajo información de variables clínicas y socioeconómicas. Una vez tomadas las muestras se analizaron las mediciones antropométricas (peso, talla, edad) y determinación del estado nutricional. El análisis estadístico se realizó utilizando el software de distribución libre: Epiinfo versión 7; a las variables cualitativas se les realizó análisis descriptivo y a las variables cuantitativas análisis de medidas de tendencia central.

Las Variables en estudio fueron: sexo, seguridad social, área de ocurrencia, casos con complicaciones y ocupación medidas a nivel nominal, edad, semanas de gestación y peso medidas a nivel de razón.

Consideraciones éticas: Este estudio se considera un análisis sin riesgo, de acuerdo con la resolución 08430 de 1993, debido a que es un estudio descriptivo retrospectivo, con registros ingresados por fuentes de notificación intrahospitalaria, en donde la información se obtuvo de las historias clínicas, no requirió consentimiento informado; sin embargo, requirió aprobación del comité de ética de la institución.

\section{RESULTADOS}

En el año 2015 en el E.S.E Hospital la Divina Misericordia de Magangué Bolívar registró el ingreso de un total de 1.876 gestantes, de las cuales un 484 fueron adolescentes, correspondiéndole a la institución una prevalencia de embarazo en adolescentes del $25,8 \%$; de estos 30 casos $6,2 \%$ presentaron complicaciones durante su gestación.

Dos casos con un $6,7 \%$ correspondieron a adolescentes tempranas, mientras que 28 casos, $93,3 \%$ fueron adolescentes tardías (Ver tabla 1).

Tabla 1: Número de Casos de Embarazo en Adolescentes por grupo de edad, E.S.E Hospital la Divina Misericordia 2015.

\begin{tabular}{cccc}
$\begin{array}{c}\text { Clasificación según } \\
\text { (OMS) }\end{array}$ & $\begin{array}{c}\text { Edades } \\
\text { según } \\
\text { (OMS) }\end{array}$ & $\begin{array}{c}\text { Total, } \\
\text { Adolescentes } \\
\text { Gestantes }\end{array}$ & $\begin{array}{c}\text { Porcentaje } \\
\text { Adolescentes } \\
\text { Gestantes }\end{array}$ \\
\hline $\begin{array}{c}\text { Adolescencia } \\
\text { Temprana } \\
\text { Adolescencia } \\
\text { Tardía }\end{array}$ & $10-14$ años & 2 & $6,7 \%$ \\
\hline \hline Total, General & $15-19$ años & 28 & $93.3 \%$ \\
\hline \hline Fuente: E.S.E Hospital la Divina Misericordia, Magangué Bolívar.
\end{tabular}

El $100 \%$ de las adolescentes que presentaron complicaciones durante su gestación pertenecían a la raza mestiza.

En el análisis descriptivo de las variables cuantitativas, se observa que el valor mínimo del peso fue de $45 \mathrm{~kg}$, mientras el máximo fue de $52 \mathrm{~kg}$, con una media de 48,03 $\pm 2,09$, respectivamente.

Así mismo por edades, el mínimo fue de 13 años, mientras la edad máxima fue de 19 años, con una media de 16,2 $\pm 1,648$, respectivamente.

Las semanas donde se evidencia mayor ingreso de gestantes al programa de control prenatal, fueron entre las semanas 16 a la 22 de la gestación (Ver tabla 2). 
Tabla 2: Características antropométricas en las adolescentes con complicaciones, E.S.E Hospital la Divina Misericordia (2015).

\begin{tabular}{|c|c|c|c|}
\hline Variables & $\begin{array}{l}\text { Valor Min. } \\
\text { Antropométrico } \\
\text { al Control } \\
\text { Prenatal } \\
\end{array}$ & $\begin{array}{c}\text { Valor } \\
\text { Antropométrico } \\
\text { al Control } \\
\text { Prenatal } \\
\end{array}$ & $\begin{array}{c}\text { Promedio } \\
\text { de Variable }\end{array}$ \\
\hline Peso (kg) & $45 \mathrm{~kg}$ & $52 \mathrm{~kg}$ & $48,03 \mathrm{~kg}$ \\
\hline $\begin{array}{c}\text { Edad (Años) } \\
\text { Semanas de }\end{array}$ & 13 Años & 19 Años & 16 Años \\
\hline $\begin{array}{r}\text { Gestación al } \\
\text { Ingreso al Control } \\
\text { Prenatal }\end{array}$ & 16 Semanas & 22 Semanas & 19 Semanas \\
\hline \multicolumn{4}{|c|}{ Total, Embarazadas Adolescentes 30} \\
\hline
\end{tabular}

Del total de adolescentes con complicaciones 16 casos, $53,3 \%$ presentaban estado civil de solteras, Unión libre, diez casos, $33,3 \%$, el estado civil del grupo de solteras, mostró el mayor porcentaje de casos, en comparación con las casadas y aquellas cuyas relaciones se observaban en unión libre marital (Ver tabla 3).

Tabla 3: Número de gestaciones en adolescentes con complicaciones, de acuerdo al estado civil, E.S.E Hospital la Divina Misericordia (2015).

\begin{tabular}{ccccccc}
\hline \hline $\begin{array}{c}\text { Estado } \\
\text { civil }\end{array}$ & $\begin{array}{c}\text { Un } \\
\text { embarazo }\end{array}$ & $\mathbf{\%}$ & $\begin{array}{c}\text { Dos } \\
\text { embarazos }\end{array}$ & $\%$ & $\begin{array}{c}\text { Total, } \\
\text { General }\end{array}$ & $\%$ \\
\hline $\begin{array}{c}\text { Casada } \\
\text { Soltera }\end{array}$ & 3 & 11,5 & 1 & 25 & $\mathbf{4}$ & 13,3 \\
$\begin{array}{c}\text { Unión } \\
\text { libre }\end{array}$ & 8 & 57,6 & 1 & 25 & $\mathbf{1 6}$ & 53,3 \\
\hline \hline $\begin{array}{c}\text { Total, } \\
\text { General }\end{array}$ & $\mathbf{2 6}$ & $\mathbf{1 0 0}$ & $\mathbf{4}$ & $\mathbf{1 0 0}$ & $\mathbf{3 0}$ & $\mathbf{1 0 0}$ \\
\hline \hline
\end{tabular}

Según área de ocurrencia las complicaciones en adolescentes obstétricas, se observaron con procedencia tanto del área urbana como del área rural, mostrando un evidente incremento en el grupo de adolescentes tardías por zona urbana (Ver Figura 1).

Figura 1: Comportamiento de las complicaciones en adolescentes obstétricas, por área de ocurrencia, E.S.E Hospital la Divina Misericordia (2015).

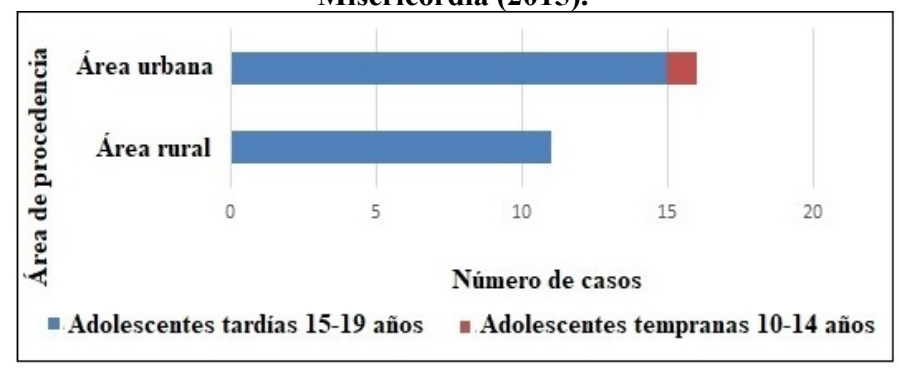

Fuente: E.S.E Hospital la Divina Misericordia

La ocupación laboral aportó 21 casos 70\%, porcentaje significativamente mayor en comparación con nueve casos $30 \%$ que sé que se encontraron al momento del estudio (Ver figura 2).
Esquema 1. Comportamiento de las complicaciones por ocupación en adolescentes obstétricas, E.S.E Hospital la Divina Misericordia (2015).

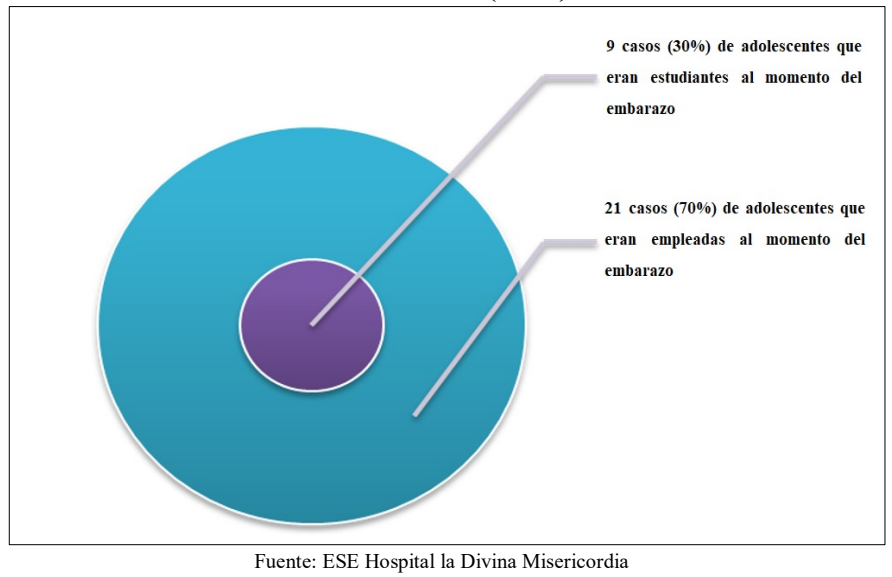

Los factores de riesgos y las complicaciones se encuentran ligadas significativamente por grupos de edades, del total de embarazos en adolescentes en edades tardías y tempranas que fueron 30 casos, las adolescentes tardías con 26 casos, 86,6\% evidenciaron complicaciones obstétricas, las más predominantes en el estudio fueron la Anemias Severas con 13 casos, $81,2 \%$ seguido de la pre eclampsia con el $100 \%$ de los casos para este grupo de edad tardío; sin embargo, las adolescentes tempranas con solo cuatro casos, $13,3 \%$ evidenciaron un menor número de complicaciones durante su gestación (Ver tabla 4).

Tabla 4: Casos con Complicaciones en Adolescentes Obstétricas de la E.S.E Hospital la Divina Misericordia (2015).

\begin{tabular}{|c|c|c|c|}
\hline Descripción & $\begin{array}{c}10 \text { - } 14 \text { años } \\
\text { (Adolescencia } \\
\text { temprana) }\end{array}$ & $\begin{array}{c}15 \text { - } 19 \text { Años } \\
\text { (Adolescencia } \\
\text { tardía) }\end{array}$ & Total \\
\hline $\begin{array}{r}\text { Amenazas de } \\
\text { aborto }\end{array}$ & 1 & 4 & 5 \\
\hline$\%$ & 20 & 80 & $100 / 16,6$ \\
\hline Anemias severas & 3 & 13 & 16 \\
\hline$\%$ & 18,7 & 81,2 & $100 / 53,3$ \\
\hline Preeclampsia & 0 & 9 & 9 \\
\hline$\%$ & 0 & 100 & $100 / 30$ \\
\hline $\begin{array}{r}\text { Total, } \\
\text { Adolescentes } \\
\text { Embarazadas }\end{array}$ & $4 / 13,3$ & $26 / 86,6$ & $30 / 100$ \\
\hline
\end{tabular}

El comportamiento de las complicaciones de las adolescentes obstétricas según nivel socioeconómico evidencia que en el estrato I las anemias severas predominaron con 14 casos, 58,3\% mientras que el estrato II las complicaciones de Pre-eclampsias, Amenazas de Abortos y anemias severas evidenciaron un comportamiento similar.

Cabe resaltar que el estrato I, aporto el mayor número de casos 24 para el $80 \%$ (Ver tabla 5 ). 
Tabla 5: Comportamiento de las Complicaciones Según Nivel Socioeconómico en Adolescentes Obstétricas de la E.S.E Hospital la Divina Misericordia (2015).

\begin{tabular}{rccccc}
\hline \hline $\begin{array}{c}\text { Complicaciones en } \\
\text { adolescentes } \\
\text { obstétricas }\end{array}$ & Estrato & $\mathbf{\%}$ & $\begin{array}{c}\text { Estrato } \\
\text { II }\end{array}$ & $\mathbf{\%}$ & Total \\
\hline \hline $\begin{array}{r}\text { amenazas de } \\
\text { aborto }\end{array}$ & 3 & 12,5 & 2 & 33,3 & $\mathbf{5}$ \\
Anemias severas & 14 & 58,3 & 2 & 33,3 & $\mathbf{1 6}$ \\
Preeclampsia & 7 & 29,1 & 2 & 33,3 & $\mathbf{9}$ \\
\hline \hline Total & $\mathbf{2 4}$ & $\mathbf{1 0 0 / 8 0}$ & $\mathbf{6}$ & $\mathbf{1 0 0 / 2 0}$ & $\mathbf{3 0}$ \\
\hline \hline & Fuente: E.S.E Hospital la Divina Misericordia & &
\end{tabular}

Las complicaciones de las adolescentes obstétricas según tipo de afiliación al Sistema General de Seguridad Social en Salud (SGSSS) registró que el Régimen Subsidiado fue el más afectado con 24 casos, $80,0 \%$; la Anemia Severa aporto mayor número de casos en el régimen subsidiado $14,58,3 \%$ en comparación con el régimen contributivo que solo aporto dos casos en el estudio, así mismo las complicaciones como Preclampsia con siete casos, 29,1\% y las Amenazas de Abortos con tres casos, $12,5 \%$, predominaron más en el régimen subsidiado en comparación con el régimen contributivo. (tabla $6)$.

Tabla 6: Comportamiento de las Complicaciones en Adolescentes Obstétricas Según Seguridad Social E.S.E Hospital la Divina Misericordia (2015).

\begin{tabular}{cccc}
\hline \hline $\begin{array}{c}\text { Complicaciones en } \\
\text { adolescentes obstétricas }\end{array}$ & Subsidiado/\% & Contributivo/\% & Total \\
\hline Amenazas de aborto & $3 / 12,5$ & $2 / 33,3$ & 5 \\
Anemias Severas & $14 / 58,3$ & $2 / 33,3$ & 16 \\
Preeclampsia & $7 / 29,1$ & $2 / 33,3$ & 9 \\
\hline \hline & $\mathbf{2 4 / 8 0}$ & $\mathbf{6 / 2 0}$ & $\mathbf{3 0}$ \\
\hline \hline
\end{tabular}

\section{DISCUSIÓN}

La ESE Hospital Divina Misericordia evidencio que la prevalencia de embarazo en adolescentes durante el año 2015 fue de un $25,8 \%$ este dato coincide con estudios internacionales $(6,7)$ donde el aumento de frecuencia de gestaciones durante la adolescencia es alto, oscilando entre 7 y $25 \%$ de los embarazos en edades reproductivas. Otros estudios internacionales describen un $15 \%$ de embarazos. $(6,7)$.

Las bajas condiciones socioeconómicas, así como la ignorancia y la pobreza constituyen factores causales muy importantes, tanto del embarazo adolescente como del inicio precoz de las relaciones sexuales. Por ello se observa cómo la fecundidad de las adolescentes no se distribuye de forma homogénea entre los diferentes estratos sociales, apreciándose mayor prevalencia en los estratos socioeconómicos I, y un menor porcentaje en el estrato II.
En Colombia se ha disminuido la tasa de fecundidad en adolescentes en zonas como Bogotá y región central, así como se observó incremento en la Orinoquia y Amazonas, en consideraciones generales la tasa de fecundidad en Colombia en comparación con otros países latinoamericanos ha bajado significativamente, aunque sigue siendo alta ya que de cinco mujeres embarazadas una es adolescente (8).

La afiliación al sistema de seguridad social, evidenció una variación importante, donde se observa que las complicaciones por anemias severas son más frecuentes en el régimen subsidiado en comparación con el contributivo.

La fecundidad adolescente en este estudio, es más alta entre clases sociales menos favorecidas, situación que coincide con el estudio de "Nacimientos en Pacientes Menores de 20 años", en el Hospital "Dr. Ramón Madariaga". Posadas, Misiones, de Asunción Paraguay (9).

Una limitación importante en este estudio, fue la obtención de registros en el nivel hospitalario, debido a la falta de organización de los datos y la no disponibilidad de un registro sistematizado de las atenciones a esta población.

\section{CONCLUSIONES}

La complicación obstétrica que evidencia un alto impacto para la población en estudio y que además propende el aumento sociodemográfico en el estrato I, fueron las anemias severas con 14 casos, $58,3 \%$.

Las adolescentes en edades tardías mostraron la frecuencia más alta de casos con 28 para el $93,3 \%$ a diferencia de las adolescentes en edades tempranas que solo presentaron dos casos con el $6,7 \%$.

Un dato significativo de este análisis, es la representatividad de casos por área de ocurrencia que presento mayor concentración de embarazos adolescentes en el área urbana en comparación con el área rural.

De acuerdo a la seguridad social, el régimen subsidiado evidenció un mayor número de casos en comparación al régimen contributivo, al mismo tiempo que se vincula proporcionalmente con el desarrollo de afecciones obstétricas en las adolescentes analizadas.

Los resultados obtenidos de este estudio demuestran que deben reinventarse las metas y las estrategias orientadas a canalizar el incremento de este evento en la población juvenil, todo esto para el fortalecimiento oportuno y reducción de factores condicionantes y predisponentes previos al embarazo en la adolescente, teniendo en cuenta las características sociodemográficas de las menores, así mismo servir de eje para mejorar las acciones de promoción y prevención en salud. 


\section{RECOMENDACIONES}

$\mathrm{Al}$ evidenciar una prevalencia de embarazo en adolescentes tan elevada en el municipio de Magangué, se deben fortalecer las acciones de demanda inducida para incorporar a las adolescentes a programas de planificación familiar efectivos.

Siendo el nivel socioeconómico un factor decisivo en el inicio de relaciones sexuales a temprana edad sin protección, se deben adelantar estrategias que propendan por la disminución de la deserción escolar y una educación comunitaria a los núcleos familiares.

Implementar acciones orientadas a reducir el riesgo de estimular las relaciones sexuales a edades tempranas, especialmente en los espacios y ámbitos sociales, culturales o recreacionales, donde interactúen las menores.

Hacer un llamado de carácter prioritario a las autoridades sanitarias del municipio para que, en conjunto con la institución hospitalaria, analicen los enfoques de riesgos aplicables a dicha población.

Garantizar que toda adolescente gestante, tenga acceso a los servicios de salud, llevando a cabo los controles prenatales para el desarrollo normal del neonato y así evitar las complicaciones antes mencionadas a la gestante, por otra parte, hacer énfasis en la educación temática de la sexualidad desde el componente familiar hasta el nivel educativo.

Conflicto de Interés: Los autores no declaran conflicto de interés.

Agradecimientos: E.S.E Hospital La Divina Misericordia.

\section{REFERENCIAS}

1. United Nations Children's Fund y Plan International . Vivencias y relatos sobre el embarazo en adolescentes. Informe Final de UNICEF; Panamá: Plan y UNICEF; 2014

2. HolaDoctor.com. Embarazo en la adolescencia [internet]. HolaDoctor.com; 2011 [actualizada 2019; acceso 20 agosto 2018`] Disponible en https://holadoctor.com/es/adam/embarazo-en-laadolescencia

3. United Nations Children's Fund. Estado mundial de la infancia 2011. La adolescencia: Una época de oportunidades. UNICEF; Nueva York: UNICEF; 2011.

4. Organización Mundial de la Salud. Embarazo en adolescentes: un problema culturalmente complejo. Boletín OMS. 2009;87:405484.

5. Issler JR. Embarazo en la adolescencia. Revista de Posgrado de la Cátedra Vía Medicina 2001;107:11-23.

6. Carreón VJ, Mendoza SH, Pérez HC, Gil AI, Soler HE, González SR. Factores socioeconómicos asociados al embarazo en adolescentes. Archivos de medicina Familiar.2004;6(3):70-73.

7. Herrera JA, Ersheng G, Shahabuddin AKM, Lixia D, Wei Y, Faisal M, et al. Evaluación periódica del riesgo biopsicosocial prenatal en la predicción de las complicaciones maternas y perinatales en Asia 2002-2003. Colombia Médica.2006;37(2):614.

8. Francois KE, Foley MR. Antepartum and postpartum hemorrhage. In: Gabbe SG, Niebyl JR, Simpson JL, eds. Obstetrics - Normal and Problem Pregnancies. 5th ed. Philadelphia, Pa: Elsevier Churchill Livingstone; 2007

9. Mayer ML., Barbosa SB., Benítez SM., Fochesatto NA. Nacimientos en Pacientes Menores de 20 Años, en el Hospital Dr. Ramón Madariaga. Posadas, Misiones. Revista de Posgrado de la VIa Cátedra de Medicina. 2006;161:1-4. 